\title{
Midgut expression of immune-related genes in Glossina palpalis gambiensis challenged with Trypanosoma brucei gambiense
}

\author{
Illiassou Hamidou Soumana' ${ }^{1}$ Bernadette Tchicaya ${ }^{1}$, Paul Chuchana ${ }^{2}$ and Anne Geiger ${ }^{1}$ * \\ 1 IRD-CIRAD, UMR 177, Montpellier, France \\ 2 Inserm, U844, Hôpital Saint-Eloi, Montpellier, France
}

\section{Edited by:}

Suleyman Yazar, Erciyes University, Turkey

\section{Reviewed by:}

Marc S. Dionne, King's College London, UK

Ravi Durvasula, University of New Mexico School of Medicine, USA

\section{${ }^{*}$ Correspondence:}

Anne Geiger, IRD-CIRAD, UMR 177, CIRAD TA A-17/G, Campus International de Baillarguet, 34398 Montpellier, Cedex 5, France e-mail: anne.geiger@ird.fr
Tsetse flies from the subspecies Glossina morsitans morsitans and Glossina palpalis gambiensis, respectively, transmit Trypanosoma brucei rhodesiense and Trypanosoma brucei gambiense. The former causes the acute form of sleeping sickness, and the latter provokes the chronic form. Although several articles have reported G. m. morsitans gene expression following trypanosome infection, no comparable investigation has been performed for $G$. p. gambiensis. This report presents results on the differential expression of immune-related genes in G. p. gambiensis challenged with T. b. gambiense. The aim was to characterize transcriptomic events occurring in the tsetse gut during the parasite establishment step, which is the crucial first step in the parasite development cycle within its vector. The selected genes were chosen from those previously shown to be highly expressed in G. m. morsitans, to allow further comparison of gene expression in both Glossina species. Using quantitative PCR, genes were amplified from the dissected midguts of trypanosome-stimulated, infected, non-infected, and self-cleared flies at three sampling timepoints $(3,10$, and 20 days) after a bloodmeal. At the 3 -day sampling point, transferrin transcripts were significantly up-regulated in trypanosomechallenged flies versus flies fed on non-infected mice. In self-cleared flies, serpin-2 and thioredoxin peroxidase-3 transcripts were significantly up-regulated 10 days after trypanosome challenge, whereas nitric oxide synthase and chitin-binding protein transcripts were up-regulated after 20 days. Although the expression levels of the other genes were highly variable, the expression of immune-related genes in G. p. gambiensis appears to be a time-dependent process. The possible biological significance of these findings is discussed, and the results are compared with previous reports for G. m. morsitans.

Keywords: Glossina palpalis gambiensis, Trypanosoma brucei gambiense infection, midgut, immune gene expression

\section{INTRODUCTION}

Tsetse flies (Glossina sp.) are responsible for the cyclical transmission of protozoan known as trypanosomes, which are the causative agents of Human African Trypanosomiasis (HAT; or sleeping sickness) and Animal African Trypanosomiasis (or nagana) throughout sub-Saharan Africa (Simarro et al., 2003). It is estimated that 60 million people in 36 African countries are at risk of HAT (WHO, 2006). Sleeping sickness is fatal if untreated (Holmes, 2013). No vaccine is available for the mammalian host, as the variant surface glycoprotein (VSG) coating the trypanosome plasma membrane makes the development of a vaccine unlikely. Furthermore, part of this VSG composition and structure periodically varies, which in turn causes periodic antigenic variations that allow the trypanosome to escape both injected and/or natural host-produced antibodies. Finally, this coat prevents antibodies from gaining access to invariant surface molecules (MacGregor et al., 2012). To complicate matters, chemotherapy treatments have major harmful side effects and are difficult to administer (Priotto et al., 2008), and the emergence of parasite resistance has decreased the efficacy of drug treatments (Baker et al., 2013).

The fly vector, which is strictly hematophagous, acquires the parasite during a bloodmeal on an infected host, whether human or animal. To be transmitted, trypanosomes must first establish in the midgut; then they migrate to the salivary glands, where they mature into an infective metacyclic form; they are finally secreted in the saliva during a bloodmeal (Hu and Aksoy, 2006).

In ideal laboratory conditions, $40 \%$ or more of challenged flies will eliminate their ingested trypanosomes (Lehane et al., 2003, 2008). For field flies, infection rates rarely exceed $10 \%$ of the population (Frézil and Cuisance, 1994). Only a small number of flies are able to transmit parasites to a host (Aksoy et al., 2003; Rio et al., 2004). Approximately $72 \mathrm{~h}$ following ingestion of the infected bloodmeal, a process of attrition leads to the complete elimination of the infection in a high proportion of flies, whereas parasites are established in the gut during a successful infection. Flies can be arranged into two groups following this 
attrition process: those that are susceptible to infection when trypanosomes are detectable in the fly's gut, and those that are refractory (or self-cleared) when trypanosomes are undetectable (Gibson and Bailey, 2003). Differential expression of midgut effector molecules in different tsetse species or strains may account for the variability in susceptibility to trypanosomes (Haddow et al., 2005). Many other factors are involved in determining the success or failure of the infection and maturation processes (Maudlin and Welburn, 1994). These include fly sex, age, and nutritional status at the time of exposure to infectious trypanosomes (Welburn and Maudlin, 1992); antimicrobial peptides (AMPs; Hao et al., 2001; Hu and Aksoy, 2006); trypanosomebinding lectins (Maudlin and Welburn, 1988; Welburn etal., 1994); gut-associated EP protein (Chandra et al., 2004; Haines et al., 2005, 2010); and reactive oxygen species (ROS; Hao et al., 2003).

Since trypanosomes initiate their cycle within the host midgut, an improved understanding of the differential expression of immunity genes could provide opportunities to identify genes possibly involved in tsetse refractoriness, as well as those involved in active infections.

Previously, Lehane et al. (2003) reported a number of selected genes (including genes related to fly immunity) that exhibit altered expression patterns in response to trypanosome infection, during their establishment in the fly gut. These studies were conducted on insectary-maintained flies belonging to the subspecies Glossina morsitans morsitans (initially collected in Zimbabwe) and challenged with Trypanosoma brucei brucei. In east African countries, flies of the morsitans group transmit trypanosomes belonging to the subspecies Trypanosoma brucei rhodesiense, causing the acute form of sleeping sickness. Conversely, Glossina palpalis gambiensis (palpalis group) flies in West Africa transmit trypanosomes belonging to the subspecies Trypanosoma brucei gambiense, causing the chronic form of the disease (Hoare, 1972). We chose to investigate G. p. gambiensis challenged with T. b. gambiense, as this approach had previously not been utilized to examine this specific Glossina/trypanosome couple. Furthermore, our choice enables checking whether the responses of the two Glossina subspecies to their, respectively, transmitted trypanosome subspecies are comparable or not. We investigated the G. p. gambiensis response at the trypanosome invasion step, as it is determinant in whether the parasite will establish within the fly gut (i.e., flies susceptible to trypanosome infection) or if it will be eliminated (i.e., flies refractory to trypanosome infection, or self-cleared/self-cured flies). Finally, we investigated 12 immune genes selected from those previously reported to be highly overexpressed in G. m. morsitans challenged with T. b. brucei (Lehane et al., 2003).

\section{MATERIALS AND METHODS ETHICS STATEMENT}

All experiments on animals were conducted according to internationally recognized guidelines. The experimental protocols were approved by the Ethics Committee on Animal Experiments and the Veterinary Department of the Centre International de Recherche Agronomique pour le Développement (CIRAD), Montpellier, France.

\section{T. b. gambiense STRAIN AND FLY INFECTION}

The T. $b$. gambiense isolate $\mathrm{S} 7 / 2 / 2$ used for fly infections was isolated in 2002 by rodent inoculation from a HAT patient detected in the sleeping sickness focus of Bonon, Côte d'Ivoire (Ravel et al., 2006).

Female G. p. gambiensis tsetse flies were collected from the CIRAD Baillarguet insectary. Following adult emergence, the population was maintained in a level 2 containment insectary at $23^{\circ} \mathrm{C}$ and $80 \%$ relative humidity (Geiger et al., 2005). This fly colony originated from Burkina Faso, where it was first collected 40 years ago.

A T. b. gambiense stabilate was thawed at room temperature and $0.2 \mathrm{ml}$ was injected intraperitoneally into Balb/cj mice. To monitor infection, tail blood was examined using a phasecontrast microscope at $400 \times$ magnification. Teneral flies (less than $32 \mathrm{~h}$ old) were fed on the abdomens of infected mice (30 flies per mouse, on average); mice displayed parasitemia levels between 16 and $64 \times 10^{6}$ parasites $/ \mathrm{ml}$, as determined by the matching method (Herbert and Lumsden, 1976). Only flies that had ingested a large bloodmeal were retained for further studies. After 10-day and 20-day timepoints, anal drops were collected from flies that fed on infected mice, and their infection status was assessed. T. $b$. gambiense presence was determined by PCR of chelex-extracted anal drop DNA using the TBR1 and TBR2 primers (Moser et al., 1989). The presence of parasites in the anal drop was positive indication for midgut infection (i.e., susceptible flies). By contrast, the absence of the parasite indicated that these flies receiving an infected bloodmeal were refractory to infection. Anal drop analysis was selected in this study to determine fly contamination status since the whole midgut was later used for RNA extraction. The prevalence of midgut infection was less than $5 \%$ for 10 -day flies and greater than $10 \%$ for 20 -day flies, corresponding with recently recorded values from artificial infection experiments (Ravel et al., 2006; Hamidou Soumana et al., 2014). Using this procedure, flies were separated into infected and selfcured groups (i.e., flies that had ingested trypanosomes in their bloodmeal but had cleared the infection), and dissected according to the method described by Penchenier and Itard (1981). The 3 -day group of flies received an infected bloodmeal and was dissected 3 days later; they were compared with 3-day flies fed on an uninfected bloodmeal, considered as control flies. Dissected tsetse fly midguts were collected (pool of seven fly guts per sample) in $400 \mu \mathrm{l}$ of RNA later reagent and stored at $-80^{\circ} \mathrm{C}$ until RNA extraction.

Sampling times were chosen according to a previously determined time course of susceptible fly infection by trypanosome (Van Den Abbeele etal., 1999; Ravel etal., 2003). The 3-day and 10-day sampling times were respectively, selected to target differentially expressed genes involved in early events associated with trypanosome entry into the midgut, and the establishment of infection. The 20-day time point was selected to target genes involved in events occurring relatively late in trypanosome infection, within its vector.

\section{TOTAL RNA ISOLATION}

Midguts were dissected from 3-day flies (fed on either an infected or a non-infected bloodmeal), as well as from infected and 
self-cleared flies at 10 and 20 days after the infective bloodmeal. Each timepoint consisted of four biological replicates (seven pooled midguts). Total RNA was then extracted from each sample using Trizol reagent (Invitrogen), according to the manufacturer's specifications. RNA integrity was assessed after extraction using agarose gel electrophoresis. RNA quality and the absence of any DNA contamination were checked on an Agilent RNA 6000 Bioanalyzer and quantified using the Agilent RNA 6000 Nano kit (Agilent Technologies).

\section{IMMUNITY-RELATED GENES AND QUANTITATIVE REAL-TIME PCR PRIMERS}

Lehane etal. (2003) identified genes with putative immunerelated functions in G. m. morsitans following T. b. brucei infection. Twelve highly up-regulated genes were chosen from this study to investigate their possible differential expression in either G. p. gambiensis refractory flies versus $T$. $b$. gambiense infected flies (10- and 20-day samples), or in trypanosomestimulated flies versus control flies (3-day samples). Gene expression was measured by quantitative PCR using specific primer pairs (Table 1) designed with the PrimerBlast software (http://www.ncbi.nlm.nih.gov/tools/primer-blast/). Pairs of G. p. gambiensis tubulin beta-1 gene-specific primers were designed using the sequences from the G. $m$. morsitans tubulin beta-1 gene (GenBank accession number, DQ377071; Attardo et al., 2006).

\section{cDNA SYNTHESIS AND QUANTITATIVE REAL-TIME PCR}

Samples were treated with RNase-free TURBO DNase I (Ambion). First-strand cDNA was then synthesized from $5 \mu \mathrm{g}$ of total RNA using random hexamers and SuperScript II Reverse-Transcriptase (Invitrogen), according to the manufacturer's instructions. Quantitative PCR was performed in triplicate using $2 \mu \mathrm{l}$ of cDNA on an Mx3005P QPCR System (Agilent Technologies) and using the Brilliant II SYBR Green qPCR Kit (Agilent Technologies). The G. p. gambiensis housekeeping gene tubulin beta-1 was used as the reference gene to calculate the normalization of the relative quantification of expression. Cycle thresholds $(\mathrm{Ct})$ for each reaction were obtained using the MxPRO QPCR Software (Agilent Technologies). PCR conditions were as follows: $94^{\circ} \mathrm{C}$ for $5 \mathrm{~min}$ ( 1 cycle); $94^{\circ} \mathrm{C}$ for $45 \mathrm{~s}, 60^{\circ} \mathrm{C}$ for $45 \mathrm{~s}$, and $72^{\circ} \mathrm{C}$ for $1 \mathrm{~min}(39$ cycles); and $72^{\circ} \mathrm{C}$ for $10 \mathrm{~min}$ ( 1 cycle). The amplification efficiency was checked by the standard curve method, and melting curve analysis was performed to check PCR specificity. Relative quantification was calculated using the $2^{-\Delta \Delta C(t)}$ method (Livak and Schmittgen, 2001) and was determined for a given gene with respect to the calibrator.

\section{STATISTICAL ANALYSIS}

Quantitative PCR data was analyzed using the $2^{-\Delta \Delta C(t)}$ method. Data were then normalized against the G. p. gambiensis tubulin gene to determine the consecutive gene expression levels between infected and self-cleared flies, or stimulated and naive flies, for three timepoints post-infective bloodmeal (3, 10, and 20 days). A separate Kruskal-Wallis test (Hollander and Wolfe, 1973) was used for each immune gene, with $\mathrm{R}$ statistic software (version 2.15.0) for assessing differences in transcript expression levels. The transcription level of genes was expressed as the difference in $\mathrm{Ct}$ values and $\Delta \mathrm{Ct}$ values between infected and self-cleared flies.

\section{RESULTS}

Midgut transcript responses of G. p. gambiensis were assayed using quantitative PCR amplification of selected immune-related genes at 3,10 , and 20 days after T. b. gambiense challenge. Transcription analysis of the multiple timepoints following the challenge were used to access information about the temporal kinetics of gene regulation in the host midgut response against trypanosome establishment.

\section{TRANSCRIPT VARIATION 3 DAYS AFTER FLY TRYPANOSOME CHALLENGE}

Variation in transcript expression level was assessed at the early stage of infection by comparing 3-day flies that received an infective bloodmeal with 3-day flies fed on a non-infected bloodmeal.

Table 1 | Primers of immunity-related genes designed for quantitative PCR.

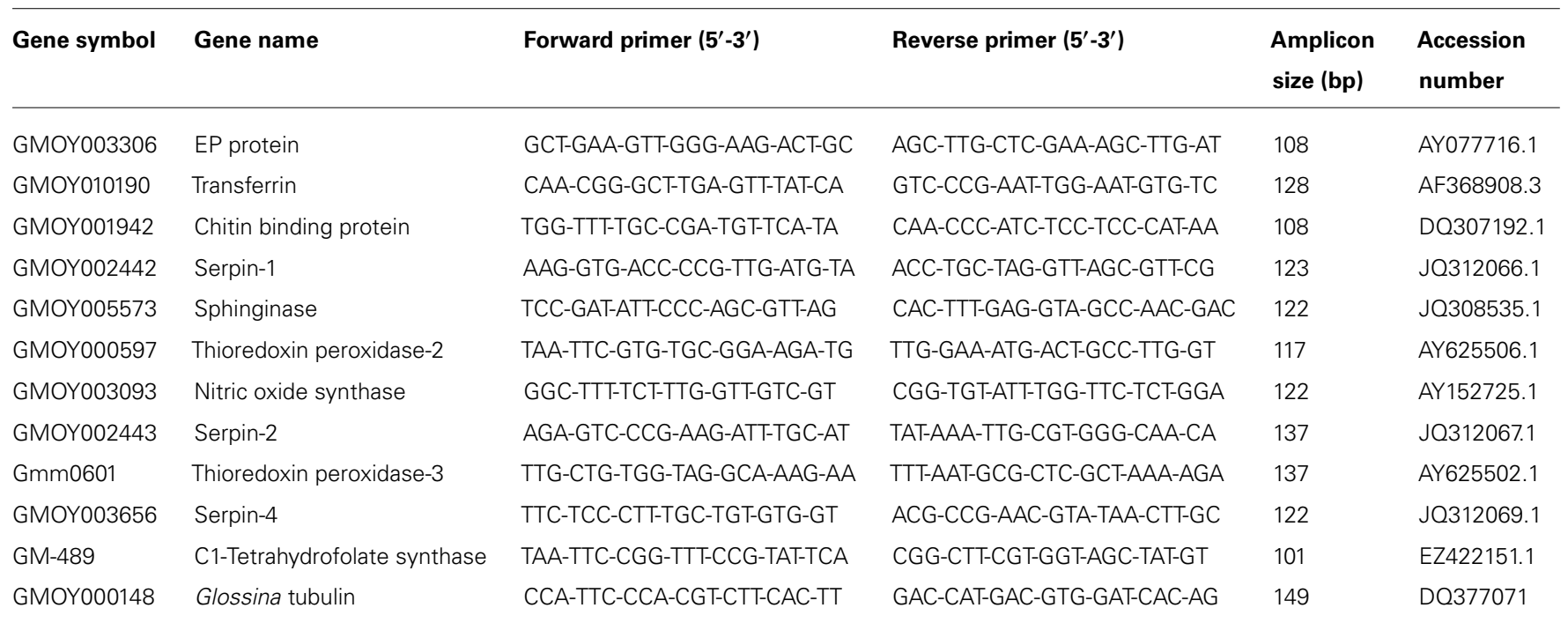


The infected bloodmeal induced a significant increase $(p<0.05)$ in transferrin transcripts 3 days after the trypanosome challenge (Figure 1A).

\section{GENE EXPRESSION IN INFECTED VERSUS SELF-CLEARED FLIES}

Comparison of infected and self-cleared flies showed that serpin-2 and thioredoxin peroxidase- 3 were expressed significantly higher $(p<0.05)$ in self-cleared flies at 10 days post-challenge with the trypanosome (Figures 1B,C, respectively). At 20 days postinfected bloodmeal, nitric oxide synthase (NOS) and chitinbinding protein were significantly overexpressed in refractory tsetse flies versus infected flies $(p<0.05$; Figures 1D,E, respectively). Most of the other selected genes displayed differences in gene expression between refractory and infected flies, although their recorded differences were not statistically significant.

\section{EXPRESSION LEVEL IN SUSCEPTIBLE FLIES THROUGHOUT THE COURSE OF THE INFECTION}

By comparing transcript levels at the three timepoints postchallenge with the parasite, we observed a decrease in the expression of chitin-binding protein transcripts along the progression of the infection $(p=0.03)$ for stimulated flies (3-days sampling timepoint) as compared to infected flies sampled 10 days post-infected bloodmeal uptake. Similar results were recorded for the chitin-binding protein transcript expression level, when comparing tsetse flies infected for 10 and 20 days $(p=0.02)$.

\section{DISCUSSION}

In the present study we investigated the expression profile of immune-related genes in G. p. gambiensis following a $T . b$. gambiense challenge. The expression level of selected genes was compared at three crucial time points of the infection process using quantitative PCR.

Twelve immune-related genes were selected on the basis of their high differential expression in the G. m. morsitans/T. $b$. brucei couple, as previously reported by Lehane et al. (2003). In the G. p. gambiensis/T. b. gambiense system, only 5 of these 12 genes displayed different expression profiles between trypanosome-challenged flies and control flies.

Nitric oxide is a signaling and immune effector molecule synthesized by the NOS (Bayne et al., 2001; Rivero, 2006). NOS production is induced in Drosophila midgut and hemocytes

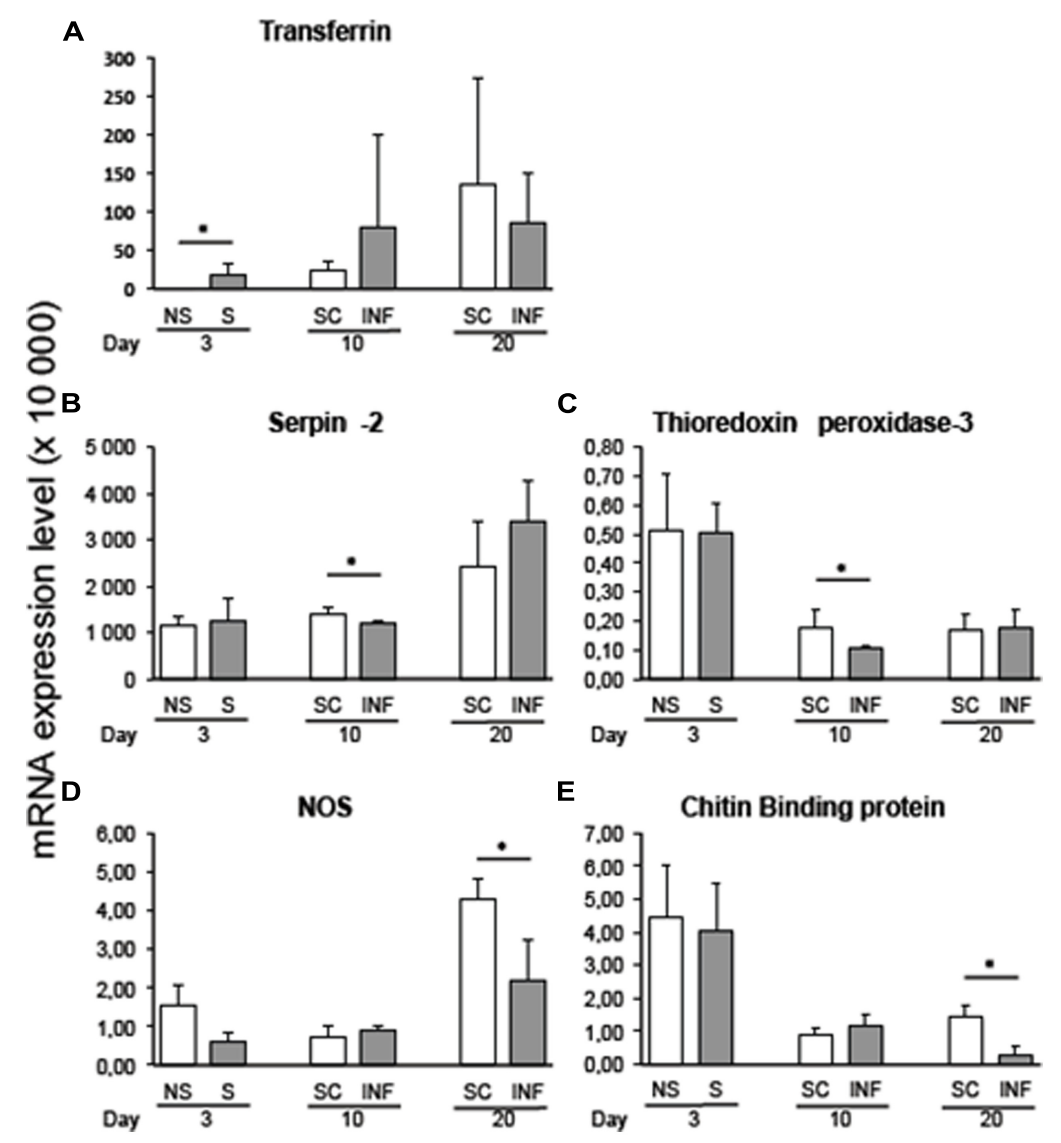

FIGURE 1 | qRT-PCR expression analysis of immune-related Glossina palpalis gambiensis genes at 3,10 , and 20 days post-challenge with Trypanosoma brucei gambiense, normalized against the
G. p. gambiensis tubulin gene. (A) Transferrin; (B) Serpin-2; (C) Thioredoxin peroxidase-3; (D) NOS; (E) Chitin binding protein. The "*" represents significant difference between infected and self-cleared samples $(p<0.05)$. 
challenged with bacteria or parasitoids; in mosquito it was described as a midgut-associated parasite antagonist that kills Plasmodium ookinetes (Peterson et al., 2007). Our results, showing a significant up-regulation of NOS transcripts in self-cleared flies at 20 days, are in agreement with those previous findings. Nitric oxide could be a part of the process leading to T. $b . \mathrm{gam}$ biense clearing in G. p. gambiensis. This has been demonstrated by injecting a specific NOS inhibitor into Drosophila body cavity prior to infection, which significantly increased parasite survival (Carton et al., 2009). However, Hao et al. (2003) showed NOS to be down-regulated by infection and not modulated by tsetse age. Its host immune response involvement may depend on the tsetse/trypanosome species couple.

Digestion of the bloodmeal can also generate ROS, which may cause damages such as enzyme inactivation, DNA degradation, and deterioration of the cellular membrane (Droge, 2002). No difference was found in the expression of thioredoxin peroxidase- 2 between infected and self-cleared flies, while thioredoxin peroxidase-3 was significantly up-regulated in 10day self-cleared flies. This enzyme may offer protection against ROS generated during the immune response (Lehane etal., 2003).

Among the three serpin (serine protease inhibitor) genes investigated, only serpin-2 was significantly over-expressed in self-cleared flies at 10 days post-challenge. The main molecular functions attributed to serpins range from the inhibition of blood coagulation to host inflammation and platelet aggregation, which are likely crucial for blood-feeding insects (Stark and James, 1995; Chmelar et al., 2011). Immune-related CLIP domain serine proteases and their inhibitors, the serpins were previously identified in G. morsitans (Mwangi et al., 2011). Serine proteases play an important role in the activation of the Toll or IMD pathways. Many serine proteases involved in the immune response exist in a fine balance with serine protease inhibitors to ensure that the impact of protease-activated cascades remains localized in time and space (Muta and Iwanaga, 1996; Jiang and Kanost, 2000). Drosophila serpin also plays a role in the regulation of Toll-mediated antifungal defense (Levashina et al., 1999; Ahmad et al., 2009). The large number of serpin transcripts found in the tsetse midgut may reflect the need to inactivate the complement and coagulation cascades of the bloodmeal, so as to protect the midgut epithelium and retain the meal in a physical state suitable for digestion. Thus, differences in serpin gene expression between the different groups of G. p. gambiensis flies fed on blood may reflect differences in their function according to the flies' status.

Surprisingly, no significant changes were found in G. p. gambiensis EP protein transcript levels at any stage of the T. $b$. gambiense infection. In G. m. morsitans, EP protein was strongly up-regulated following fly challenge with Gram-negative bacteria, as well as in response to trypanosome infection (Haines et al., 2005, 2010). Furthermore, tsetse EP protein may be involved in immune modulation, as RNAi knockdown increased susceptibility to trypanosome infection. Tsetse EP protein transcript levels are, however, dramatically reduced after 3 days of starvation (Haines et al., 2010). In our study, flies were starved for 3 days prior to dissection to remove any bloodmeal in the fly gut, which could explain the absence of variation in EP protein transcripts. Akoda et al. (2009), however, reported starvation to result in a significant reduction in non-induced baseline immune gene expression, but only after a longer starvation (4 days for newly emerged flies; 7 days for older flies).

In hematophagous insects, iron-binding protein is essential for sequestering iron, which overabundance can quickly lead to oxidative stress, a potentially destructive process for membranes, proteins, and nucleic acids. The transferrin gene expression level was significantly increased in 3-day stimulated flies versus control flies. This observation is in agreement with results reported on transferrin transcription in mosquito (Yoshiga et al., 1997) and in Bombyx mori (Yun etal., 1999). In tsetse flies and other insects, transferrin plays multiple physiological roles in immunity, iron metabolism, and reproduction, and displays tissue-dependent expression levels (Nichol et al., 2002). As shown in other insects, transferrin mRNA levels increase upon bacterial challenge in tsetse, suggesting that transferrin may play an additional role in immunity (Guz et al., 2007). In contrast, tsetse flies that had cleared the trypanosome did not show any difference in transferrin transcript levels when compared with infected flies at 10 and 20 days post-infected bloodmeal. Similar results were reported by Lehane et al. (2003) for T. b. brucei infected G. m. morsitans versus self-cleared flies. The parasite, competing in limited dietary iron environment, may modulate host gene expression.

Chitin-binding protein gene expression increased significantly in self-cleared flies 20 days after the infected bloodmeal. Chitin is the main constituent of the peritrophic membrane (PM), a physical barrier preventing trypanosome entry into the ecto-peritrophic space, and thus constitutes an obstacle to parasite establishment in the midgut. This chitin-binding gene is homologous to the Drosophila gene chit, which encodes a chitinase-like protein (Kawamura et al., 1999). In Sodalis glossinidius, the secondary symbiont of the tsetse fly that favors fly infection by trypanosomes, a homologous gene encodes a chitinase that was previously hypothesized to hydrolyze pupal chitin into glucosamine which inhibits the fly midgut lectin lethal to procyclic forms of the trypanosome (Welburn and Maudlin, 1999). Based on the trypanosome developmental cycle within the tsetse fly, one would expect the increase in chitinase gene transcripts to occur much earlier than the observed 20 days post-infected bloodmeal. In Anopheles, for example, chitin-binding protein and the enzyme involved in PM formation both displayed increased expression 3$24 \mathrm{~h}$ after the bloodmeal in all flies analyzed, independent of their infection status (Dimopoulos et al., 1998). This does, however, raise an additional question on the actual role of this protein in Anopheles.

The overall results on G. p. gambiensis immune-related genes shows their expression to be highly dependent either on the stage of trypanosome invasion and/or the status of the fly (i.e., susceptible or refractory to trypanosome infection). The midgut response represents part of the Glossina defense arsenal against trypanosomes. Nevertheless, important variability between individual tsetse fly responses to trypanosome infection was observed. This variability could be due to variation in the size of the infected bloodmeal, and in turn to the differences in the number of ingested parasites; it 
could also be due to normal biological variability in the individual host's response. In addition, significant differences were noticed between the G. p. gambiensis gut immune-related response and that displayed by G. m. morsitans following infection of the gut with $T$. $b$. brucei. These results strongly encourage broader investigations aimed at evaluating and identifying the factors causing these differences between the G. p. gambiensis and G. m. morsitans responses. Improved understanding in this domain is expected to be particularly relevant to identify common gene targets that would be suitable for controlling both forms of sleeping sickness. Transcriptional analysis is expected to provide data that are at the basis of the physiological response(s) of an organism to any perturbation. The recorded data will, in turn, provide further research directions that could consist, in a next step, in a proteomic analysis to assess, whether or not, the expressed genes are really translated into the corresponding proteins, and, finally, which role they actually play.

\section{ACKNOWLEDGMENTS}

The authors thank the "Région Languedoc-Roussillon - Appel d'Offre Chercheur d'Avenir 2011," the "Service de Coopération et d'Action Culturelle de l'Ambassade de France au Niger" and the "Institut de Recherche pour le Développement" for their financial support. Illiassou Hamidou Soumana is a Ph.D. student supported by the Embassy of France in Niger, Service de Coopération et d'Action Culturelle (SCAC).

\section{REFERENCES}

Ahmad, R., Rasheed, Z., and Ahsan, H. (2009). Biochemical and cellular toxicology of peroxynitrite: implications in cell death and autoimmune phenomenon. Immun. Immunotoxicol. 31, 388-396. doi: 10.1080/08923970802709197

Akoda, K., Van den Bossche, P., Marcotty, T., Kubi, C., Coosemans, M., De Deken, R., et al. (2009). Nutritional stress affects the tsetse fly's immune gene expression. Med. Vet. Entomol. 23,195-201. doi: 10.1111/j.1365-2915.2009.00799.x

Aksoy, S., Gibson, W. C., and Lehane, M. J. (2003). Interactions between tsetse and trypanosomes with implications for the control of trypanosomiasis. Adv. Parasitol. 53, 1-83. doi: 10.1016/S0065-308X(03)53002-0

Attardo, G. M., Strickler-Dinglasan, P., Perkin, S. A., Caler, E., Bonaldo, M. F., Soares, M. B., et al. (2006). Analysis of fat body transcriptome from the adult tsetse fly, Glossina morsitans morsitans. Insect Mol. Biol. 15, 411-424. doi: 10.1111/j.13652583.2006.00649.x

Baker, N., de Koning, H. P., Mäser, P., and Horn, D. (2013). Drug resistance in African trypanosomiasis: the melarsoprol and pentamidine story. Trends Parasitol. 29, 110-118. doi: 10.1016/j.pt.2012.12.005

Bayne, C. J., Hahn, U. K., and Bender, R. C. (2001). Mechanisms of molluscan host resistance and of parasite strategies for survival. Parasitology 123, S159-S167. doi: $10.1017 /$ S0031182001008137

Carton, F., Frey, F., and Nappi, A. J. (2009). Parasite-induced changes in Nitric Oxide levels in Drosophila paramelanica. J. Parasitol. 95, 1134-1141. doi: 10.1645/GE2091.1

Chandra, M., Liniger, M., Tetley, L., Roditi, I., and Barry, J. D. (2004). TsetseEP, a gut protein from the tsetse Glossina morsitans, is related to a major surface glycoprotein of trypanosomes transmitted by the fly and to the products of a Drosophila gene family. Insect Biochem. Mol. Biol. 34, 1163-1173. doi: 10.1016/j.ibmb.2004.07.004

Chmelar, J., Oliveira, C. J., Rezacova, P., Francischetti, I. M., Kovarova, Z., Pejler, G., et al. (2011). A tick salivary protein targets cathepsin $G$ and chymase and inhibits host inflammation and platelet aggregation. Blood 117, 736-744. doi: 10.1182/blood-2010-06-293241

Dimopoulos, G., Seeley, D., Wolf, A., and Kafatos, F. C. (1998). Malaria infection of the mosquito Anopheles gambiae activates immune-responsive genes during critical transition stages of the parasite life cycle. EMBO J. 17, 6115-6123. doi: 10.1093/emboj/17.21.6115
Droge, W. (2002). Free radicals in the physiological control of cell function. Physiol. Rev. 82, 47-96.

Frézil, J. L., and Cuisance, D. (1994). Trypanosomiasis, diseases with future: prospects and uncertainty. Bull. Soc. Pathol. Exot. 87, 391-393.

Geiger, A., Ravel, S., Frutos, R., and Cuny, G. (2005). Sodalis glossinidius (Enterobacteriaceae) and vectorial competence of Glossina palpalis gambiensis and Glossina morsitans morsitans for Trypanosoma congolense savannah type. Curr. Microbiol. 51, 35-40. doi: 10.1007/s00284-005-4525-6

Gibson, W., and Bailey, M. (2003). The development of Trypanosoma brucei within the tsetse fly midgut observed using green fluorescent trypanosomes. Kinetoplastid Biol. Dis. 2, 1-13. doi: 10.1186/1475-9292-2-1

Guz, N., Attardo, G. M., Wu, Y., and Aksoy, S. (2007). Molecular aspects of transferrin expression in the tsetse fly (Glossina morsitans morsitans). J. Insect Physiol. 53, 715-23. doi: 10.1016/j.jinsphys.2007.03.013

Haddow, J. D., Haines, L. R., Gooding, R. H., Olafson, R. W., and Pearson, T. W. (2005). Identification of midgut proteins that are differentially expressed in trypanosome-susceptible and normal tsetse flies (Glossina morsitans morsitans). Insect Biochem. Mol. Biol. 35, 425-433. doi: 10.1016/j.ibmb.2005.01.015

Haines, L. R., Jackson, A. M., Lehane, M. J., Thomas, J. M., Yamaguchi, A. Y., Haddow, J. D., et al. (2005). Increased expression of unusual EP repeat-containing proteins in the midgut of the tsetse fly (Glossina) after bacterial challenge. Insect Biochem. Mol. Biol. 35, 413-423. doi: 10.1016/j.ibmb.2005.01.005

Haines, L. R., Lehane, S. M., Pearson, T. W., and Lehane, M. J. (2010). Tsetse EP protein protects the fly midgut from trypanosome challenge. PLoS Pathog. 6:e1000793. doi: 10.1371/journal.ppat.1000793

Hamidou Soumana, I., Loriod, B., Ravel, S., Tchicaya, B., Simo, G., Rihet, P., et al. (2014). The transcriptional signatures of Sodalis glossinidius in the Glossina palpalis gambiensis flies negative for Trypanosoma brucei gambiense contrast with those of this symbiont in tsetse flies positive for the parasite: possible involvement of a Sodalis-hosted prophage in fly Trypanosoma refractoriness? Infect Genet. Evol. 24, 41-56. doi: 10.1016/j.meegid.2014.03.005

Hao, Z., Kasumba, I., and Aksoy, S. (2003). Proventriculus (cardia) plays a crucial role in immunity in tsetse fly (Diptera: Glossinidiae). Insect Biochem. Mol. Biol. 33, 1155-1164. doi: 10.1016/j.ibmb.2003.07.001

Hao, Z., Kasumba, I., Lehane, M. J., Gibson, W. C., Kwon, J., and Aksoy, S. (2001). Tsetse immune responses and trypanosome transmission: implications for the development of tsetse-based strategies to reduce trypanosomiasis. Proc. Natl. Acad. Sci. U.S.A. 98, 12648-12653. doi: 10.1073/pnas.221363798

Herbert, W. J., and Lumsden, W. H. (1976). Trypanosoma brucei: a rapid "matching" method for estimating the host's parasitemia. Exp. Parasitol. 40, 427-431. doi: 10.1016/0014-4894(76)90110-7

Hoare, C. A. (1972). The Trypanosomes of Mammals, a Zoological Monograph. Oxford: Blackwell Scientific Publications.

Hollander, M., and Wolfe, D. A. (1973). Nonparametric Statistical Methods. New York: John Wiley and Sons. 503.

Holmes, P. (2013). Tsestetransmtted trypanosomes - their biology, disease impact and control. J. Invertebr. Pathol. 112, S11-S14. doi: 10.1016/j.jip.2012.07.014

$\mathrm{Hu}, \mathrm{C}$., and Aksoy, S. (2006). Innate immune responses regulate trypanosome parasite infection of the tsetse fly Glossina morsitans morsitans. Mol. Microbiol. 60, 1194-1204. doi: 10.1111/j.1365-2958.2006.05180.x

Jiang, H., and Kanost, M. R. (2000). The clip-domain family of serine proteinases in arthropods. Insect Biochem. Mol. Biol. 30, 95-105. doi: 10.1016/S09651748(99)00113-7

Kawamura, K., Shibata, T., Saget, O., Peel, D., and Bryant, P. J. (1999). A new family of growth factors produced by the fat body and active on Drosophila imaginal disc cells. Development 126, 211-219.

Lehane, M. J., Aksoy, S., Gibson, W., Kerhornou, A., Berriman, M., Hamilton, J., et al. (2003). Adult midgut expressed sequence tags from the tsetse fly Glossina morsitans morsitans and expression analysis of putative immune response genes. Genome Biol. 4, R63. doi: 10.1186/gb-2003-4-10-r63

Lehane, M. J., Gibson, W., and Lehane, S. M. (2008). Differential expression of fat body genes in Glossina morsitans morsitans following infection with Trypanosoma brucei brucei. Int. J. Parasitol. 38, 93-101. doi: 10.1016/j.ijpara.2007.06.004

Levashina, E. A., Langley, E., Green, C., Gubb, D., Ashburner, M., Hoffmann, J. A., et al. (1999). Constitutive activation of toll-mediated antifungal defense in serpindeficient Drosophila. Science 285, 1917-1919. doi: 10.1126/science.285.5435.1917

Livak, K. J., and Schmittgen, T. D. (2001). Analysis of relative gene expression data using real-time quantitative PCR and the 2- $\Delta \Delta \mathrm{C}(\mathrm{t})$ method. Methods 25, 402-408. doi: 10.1006/meth.2001.1262 
MacGregor, P., Szöoõr, B., Savill, N. J., and Matthews, K. R. (2012). Trypanosomal immune evasion, chronicity and transmission: an elegant balancing act. Nat. Rev. Microbiol. 10, 431-438. doi: 10.1038/nrmicro2779

Maudlin, I., and Welburn, S. C. (1988). The role of lectins and trypanosome genotype in the maturation of midgut infections in Glossina morsitans. Trop. Med. Parasitol. 39, 56-58.

Maudlin, I., and Welburn, S. C. (1994). Maturation of trypanosome infections in tsetse. Exp. Parasitol. 79, 202-205. doi: 10.1006/expr.1994.1081

Moser, D. R., Cook, G. A., Ochs, D. E., Bailey, C. P., McKane, M. R., and Donelson, J. E. (1989). Detection of Trypanosoma congolense and Trypanosoma brucei subspecies by DNA amplification using the polymerase chain reaction. Parasitology 99, 57-66. doi: 10.1017/S0031182000061023

Muta, T., and Iwanaga, S. (1996). The role of hemolymph coagulation in innate immunity. Curr. Opin. Immunol. 8, 41-47. doi: 10.1016/S0952-7915(96)8 0103-8

Mwangi, S., Murungi, E., Jonas, M., and Christoffels, A. (2011). Evolutionary genomics of Glossina morsitans immune-related CLIP domain serine proteases and serine protease inhibitors. Infect. Genet. Evol. 11, 740-745. doi: 10.1016/j.meegid.2010.10.006

Nichol, H., Law, J. H., and Winzerling, J. J. (2002). Iron metabolism in insects. Ann. Rev. Entomol. 47, 535-559. doi: 10.1146/annurev.ento.47.091201. 145237

Penchenier, L., and Itard, J. (1981). Une nouvelle technique de dissection rapide des glandes salivaires et de l'intestin de glossines. Cah. O.R.S.T.O.M. Serie Ent. méd. et Parasitol. 19, 55-57.

Peterson, T. M., Gow, A. J., and Luckhart, S. (2007). Nitric oxide metabolites induced in Anopheles stephensi control malaria parasite infection. Free Radical Biol. Med. 42, 132-142. doi: 10.1016/j.freeradbiomed.2006.10.037

Priotto, G., Pinoges, L., Fursa, I. B., Burke, B., Nicolay, N., Grillet, G., et al. (2008). Safety and effectiveness of first line eflornithine for Trypanosoma brucei gambiense sleeping sickness in Sudan: cohort study. BMJ 336, 705-708. doi: 10.1136/bmj.39485.592674.BE

Ravel, S., Grébaut, P., Cuisance, D., and Cuny, G. (2003). Monitoring the developmental status of Trypanosoma brucei gambiense in the tsetse fly by means of PCR analysis of anal and saliva drops. Acta Trop. 88, 161-165. doi: 10.1016/S0001-706X(03)00191-8

Ravel, S., Patrel, D., Koffi, M., Jamonneau, V., and Cuny, G. (2006). Cyclical transmission of Trypanosoma brucei gambiense in Glossina palpalis gambiensis displays great differences among field stocks isolates. Acta Trop. 100, 151-156. doi: 10.1016/j.actatropica.2006.09.011

Rio, R. V., Hu, Y., and Aksoy, S. (2004). Strategies for the home team: symbiosis exploited for vector-borne disease control. Trends Microbiol. 12, 325-336. doi: 10.1016/j.tim.2004.05.001

Rivero, A. (2006). Nitric oxide: an antiparasitic molecule of invertebrates. Trends Parasitol. 22, 219-225. doi: 10.1016/j.pt.2006.02.014
Simarro, P. P., Louis, F. J., and Jannin, J. (2003). Sleeping sickness, forgotten illness: what are the consequences in the field? Med. Trop. 63, 231-235.

Stark, K. R., and James, A. A. (1995). A factor Xa-directed anticoagulant from the salivary glands of the yellow fever mosquito Aedes aegypti. Exp. Parasitol. 81, 321-331. doi: 10.1006/expr.1995.1123

Van Den Abbeele, J., Claes, Y., van Bockstaele, D., Le Ray, D., and Coosemans, M. (1999). Trypanosoma brucei spp. development in the tsetse fly: characterization of the post-mesocyclic stages in the foregut and proboscis. Parasitology 118, 469-478. doi: 10.1017/S0031182099004217

Welburn, S. C., and Maudlin, I. (1992). The nature of the teneral state in Glossina and its role in the acquisition of trypanosome infection in tsetse. Ann. Trop. Med. Parasitol. 86, 529-536.

Welburn, S. C., and Maudlin, I. (1999). Tsetse-trypanosome interactions: rites of passage. Parasitol. Today 15, 399-403. doi: 10.1016/S0169-4758(99)01512-4

Welburn, S. C., Maudlin, I., and Molyneux, D. H. (1994). Midgut lectin activity and sugar specificity in teneral and fed tsetse. Med. Vet. Entomol. 8, 81-87. doi: 10.1111/j.1365-2915.1994.tb00391.x

WHO. (2006). Human African trypanosomiasis (sleeping sickness): epidemiological update. Wkly. Epidemiol. Rec. 81, 71-80.

Yoshiga, T., Hernandez, V. P., Fallon, A. M., and Law, J. H. (1997). Mosquito transferrin, an acute-phase protein that is up-regulated upon infection. Proc. Nat. Acad. Sci. U.S.A. 94, 12337-12342. doi: 10.1073/pnas.94.23.12337

Yun, E. Y., Kang, S. W., Hwang, J. S., Goo, T. W., Kim, S. H., Jin, B. R., et al. (1999). Molecular cloning and characterization of a cDNA encoding a transferrin homolog from Bombyx mori. Biol. Chem. 380, 1455-1459. doi: 10.1515/BC.1999.188

Conflict of Interest Statement: The authors declare that the research was conducted in the absence of any commercial or financial relationships that could be construed as a potential conflict of interest.

Received: 26 July 2014; accepted: 26 October 2014; published online: 10 November 2014.

Citation: Hamidou Soumana I, Tchicaya B, Chuchana P and Geiger A (2014) Midgut expression of immune-related genes in Glossina palpalis gambiensis challenged with Trypanosoma brucei gambiense. Front. Microbiol. 5:609. doi: 10.3389/fmicb.2014. 00609

This article was submitted to Microbial Immunology, a section of the journal Frontiers in Microbiology.

Copyright (C) 2014 Hamidou Soumana, Tchicaya, Chuchana and Geiger. This is an open-access article distributed under the terms of the Creative Commons Attribution License (CC BY). The use, distribution or reproduction in other forums is permitted, provided the original author(s) or licensor are credited and that the original publication in this journal is cited, in accordance with accepted academic practice. No use, distribution or reproduction is permitted which does not comply with these terms. 